\section{Fatal exacerbation of Chad0x1-nCoV-19-induced thrombotic thrombocytopenia syndrome after initial successful therapy with intravenous immunoglobulins - a rational for monitoring immunoglobulin G levels}

The present report describes a vaccine-induced thrombotic thrombocytopenia (VITT) case with fatal exacerbation after initial improvement following initial intravenous immunoglobulin (IVIg) administration and anticoagulation. An 83-year-old woman presented at the emergency room with an alteration of her general condition. She presented with symptoms of weakness, nausea, vomiting, weight loss and spontaneous bruises without any obvious reason, 14 days after having received her first dose of ChadOx1 nCov-19. According to our medical records, she did not receive heparin or derivative during the previous 4 months. Clinical examination unraveled bruising on the upper limbs. Computer tomography (CT) of thorax and abdomen was normal. Oxygen saturation was $98 \%$ at admission and the patient was tested negative for SARS-CoV-2 infection as assessed by reverse-transcriptase polymerase chain reaction (RT-PCR). The initial laboratory investigations on the day of admission revealed that the patient was suffering from marked thrombocytopenia (i.e., platelet count of 10,000 per $\mathrm{mm}^{3}$ ), dramatically increased D-dimers plasma levels (i.e., > 20,000 ng/mL) and slightly low plasma fibrinogen (i.e., $179 \mathrm{mg} / \mathrm{dL}$ ) (Figure 1). She was transfused with a platelet concentrate (roughly $3.5 \times 10^{11}$ platelets) on the day of admission.

During the night, she suffered from dyspnea grade NYHA 4. Pulmonary ventilation and perfusion (V/O) scan was performed and disclosed bilateral pulmonary embolism. Anti-PF4 immunoglobulin G (IgG) antibodies (i.e. $1.80 \mathrm{AU} / \mathrm{mL}$, Figure 1) were detected on day 1 postadmission using a PF4/polyvinylsulfonate rapid assay (HemosIL $^{\circledR}$ AcuStar HIT IgG assay, Instrumentation Laboratory Belgium NV, Zaventem, Belgium). The diagnosis of VITT was confirmed using a heparin-induced multi-electrode aggregometry method. ${ }^{1}$ In face of the clinical picture, i.e., thrombocytopenia and thrombosis, with the presence of anti-PF4 antibodies and positive platelet activation tests within 30 days after vaccination with ChadOx1 nCov-19, VITT was diagnosed. ${ }^{2}$ The patient therefore promptly received 15 grams of IVIg (Privigen ${ }^{\circledR}$, CSL Behring Gmbh, Marburg, Germany) and methylprednisolone $1 \mathrm{mg} / \mathrm{kg}$. A second platelet concentrate (roughly $4.5 \times 10^{11}$ platelets) was administered to allow initiation of anticoagulation as the platelet count was still below 30,000 per $\mathrm{mm}^{3}$. The platelet count rapidly improved, i.e., 53,000 per $\mathrm{mm}^{3}$, and anticoagulation was started with fondaparinux $5 \mathrm{mg}$ once a day (od) subcutaneously from day 1 to day 3 (taking into account renal failure, i.e., Cockcroft-Gault creatinine clearance $<50 \mathrm{~mL} / \mathrm{min}$ ). She received additional IVIg on day 2 and 3 , at the dose of 60 grams per day for a total IVIg dose of 135 grams corresponding to 1.7 grams of IVIg per $\mathrm{kg}$ administered over a period of 48 hours. Fondaparinux dose was increased to $7.5 \mathrm{mg}$ od from day 4 to day 11 since renal function improved. On day 6 , the patient was stabilized, and her global health status was improved as witnessed by normalized platelet count, decrease in $\mathrm{D}$ dimers (i.e., from $>20,000 \mathrm{ng} / \mathrm{mL}$ at admission to 14,380 $\mathrm{ng} / \mathrm{mL}$ ) and C-reactive protein (CRP) (from $145 \mathrm{mg} / \mathrm{dL}$ at admission to $23 \mathrm{mg} / \mathrm{dL}$ ). Later that day, however, oxygen saturation dropped below $80 \%$. Cough with sputum production was noted and exacerbation of COPD with Moraxella catarrhalis infection was diagnosed. Oxygen

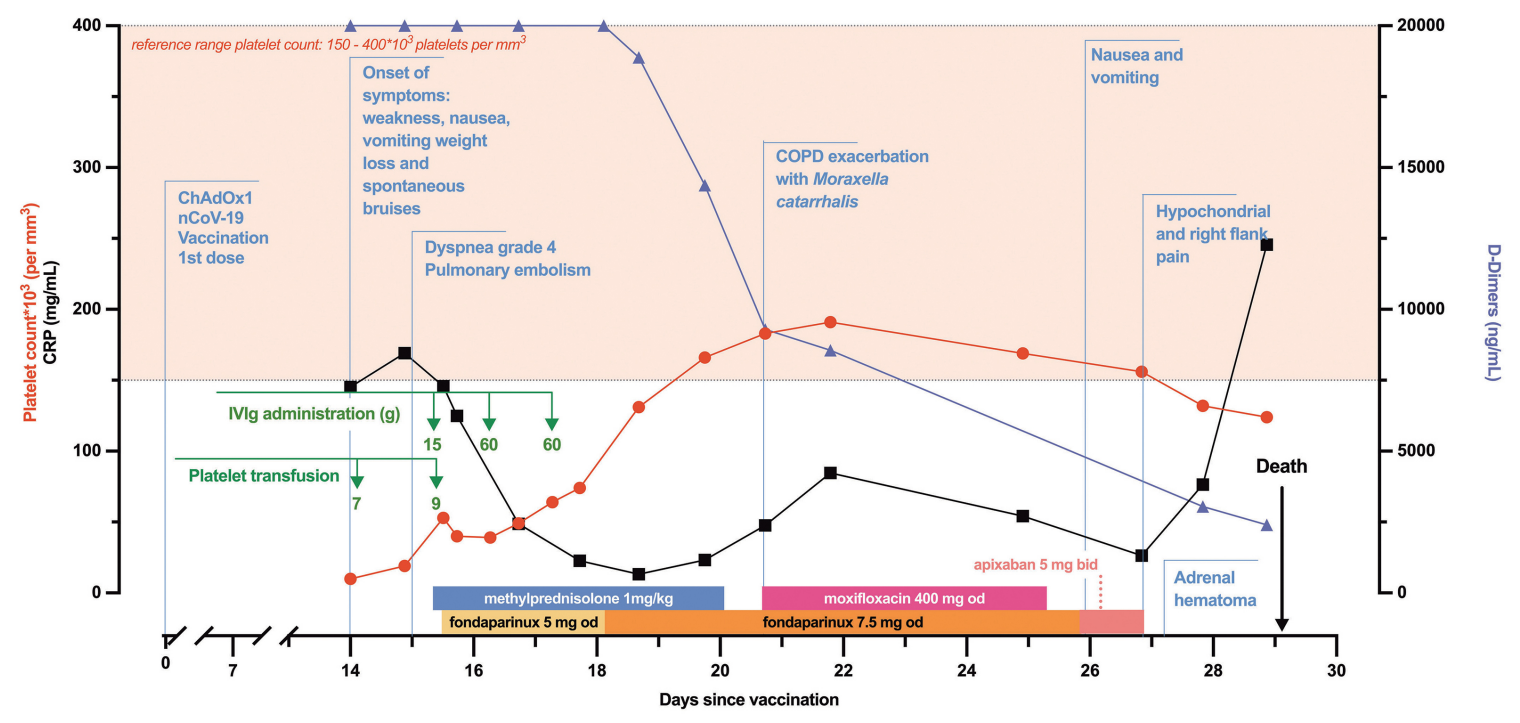

\begin{tabular}{|c|c|c|c|c|c|c|c|c|}
\hline Fibrinogen (mg/dL) & 179 & 154 & 65 & 172 & $269 \quad 433$ & 619 & 601 & Ref. range: $180-400$ \\
\hline Anti-PF4 - LIFECODES PF4 IgG (OD) & & 3.34 & 3.33 & & 3.20 & & 3.52 & Pos. if $>0.40$ \\
\hline Anti-PF4 - HemosIL ${ }^{\circledR}$ AcuStar HIT IgG (AU/mL) & & 1.80 & 1.34 & & & 0.76 & 1.25 & Pos. if $>1.00$ \\
\hline PF4-SRA (\% platelet activation w/o heparin) & & 90 & 35 & & 36 & & 79 & Pos. if $>20$ \\
\hline Immunoglobulin G (g/L) & & 6.8 & 37.1 & & 23.8 & & 11.3 & Ref. range: 7.0 - 16.0 \\
\hline
\end{tabular}

Figure 1. Clinical and laboratory data of the case. CRP: C-reactive protein; CODP: chronic obstructive pulmonary disease; HIT: heparin-induced thrombocytopenia; od: once daily. 


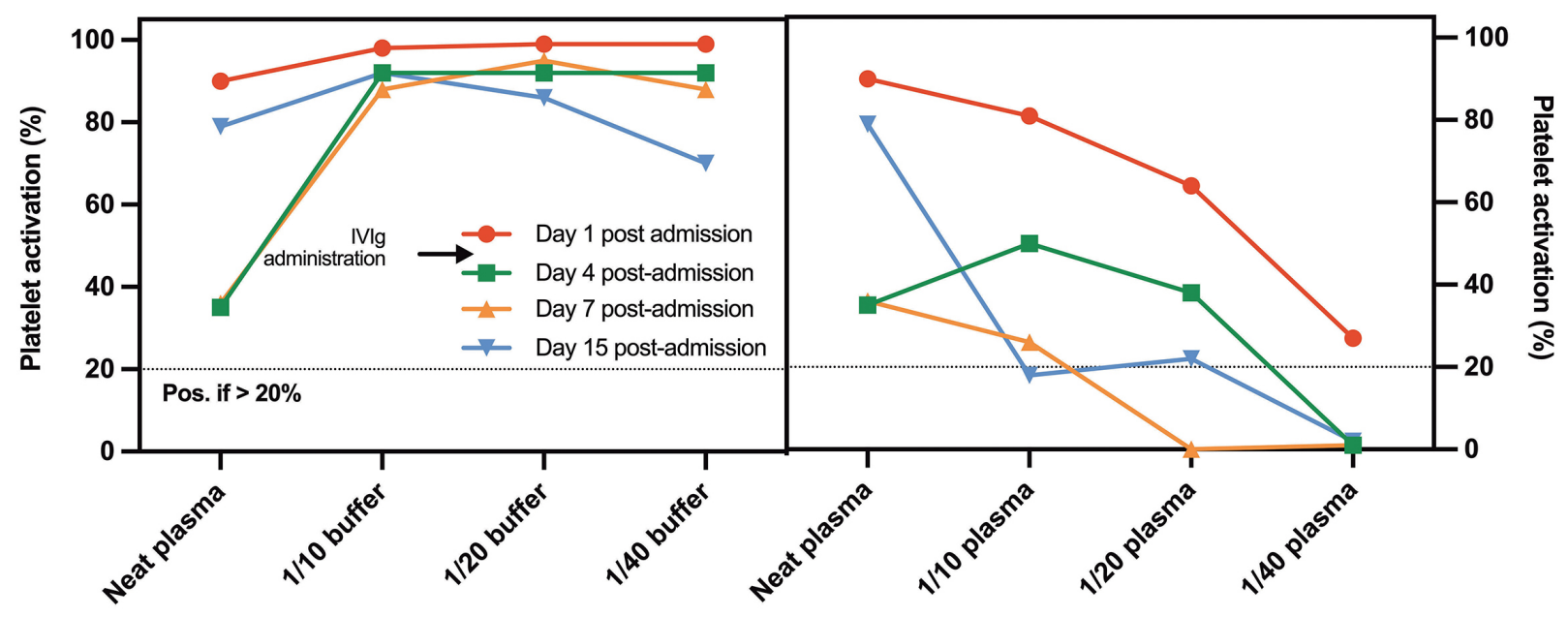

Figure 2. Dilution experiments on samples collected at day 1 post-admission (diagnosis), days 4 post-admission (after IVlg administration), day 7 post-admission (marked clinical improvement with platelet count normalization) and day 15 post-admission (deterioration of patient's status and death). Dilutions were made in normal heated plasma (containing normal immunoglobulin G [lgG] level) or in modified Tyrode's buffer at 1/10, 1/20,1/40 dilution ratios. Platelet activation was assessed without heparin in platelet-activating anti-platelet factor 4-serotonin-release assay (PF4-SRA) and results are expressed in percentage of serotonin release. IVIG: intravenous immunoglobulin.

supplementation was then started (2 liters per minute) combined with oral moxifloxacin $400 \mathrm{mg}$ od for 5 days. On day 12 post-admission, anticoagulation was switched from fondaparinux to apixaban $5 \mathrm{mg}$ twice a day (bid).

Unfortunately, the clinical status worsened on day 12 post-admission with a de novo reduction of platelet count. Abdominal CT scan showed right adrenal hematoma with left adrenal infiltrate, which is a usual presentation of adrenal infarction, as described in autoimmune heparin-induced thrombocytopenia and also recently in VITT. ${ }^{4,5}$ Four units of $500 \mathrm{IU} / \mathrm{mL}$ of prothrombin complex concentrate were administered on day 14 as an attempt to control the adrenal hematoma, but she died later that day from hypovolemic shock probably secondary to adrenal hemorrhage. A causal adrenal infarction may have existed but could not be confirmed as neither an injected CT scan nor an autopsy was performed. Nevertheless, adrenal insufficiency was not documented and cortisol levels on day 14 was still in the upper range (i.e., $21 \mu \mathrm{g} / \mathrm{dL}$, normal range: 6.2-18 $\mu \mathrm{g} / \mathrm{dL})$. Moreover, it must be noted that although apixaban was last administered on day 13 in the morning and was never reintroduced, its plasma level on day 14 was $353 \mathrm{ng} / \mathrm{mL}$ (usual Ctrough range: 22-177 $\mathrm{ng} / \mathrm{mL}$ ) and was still $132 \mathrm{ng} / \mathrm{mL}$ on day 15 . The accumulation of apixaban may thus have contributed to, or even triggered, this bleeding event. The initial infusion of platelet concentrates may also have contributed to disease progression, as well as to the pulmonary embolism observed at diagnosis. An immediate treatment with IVIg, as now recommended by the American Society of Hematology, ${ }^{6}$ could have been beneficial but the presence of active bruising, the absence of documented thrombosis and the marked thrombocytopenia guided our therapeutic choice at that time. This case further highlights how VITT is a dynamical condition, which should not be discounted in a recently vaccinated patient with only thrombocytopenia and increased D-dimer levels, even in the absence of documented thrombosis, and that IVIg should be considered promptly, along with anti-PF4 testing and thrombosis screening.
The rapid fatal outcome, occurring 2 weeks after VITT diagnosis while an improvement was noticed, raised the question of an early relapse or an exacerbation of the initial event. In an attempt to understand the possible cause(s), additional laboratory investigations were performed, as reported in Figure 1. Blood samples collected post-admission on day 1 (diagnosis), day 4 (after IVIg administration), day 7 (marked clinical improvement with platelet count normalization) and day 15 (deterioration of patient's status, leading to death) were selected to assess time-related changes of anti-PF4 antibodies, platelet activation with PF4-SRA ${ }^{7}$ and total IgG levels.

Result of the enzyme-linked immunosorbent assay (ELISA) with immobilized PF4/PVS complexes (LIFECODES PF4 IgG, Immucor Lifecodes, Jette, Belgium) remained strongly positive during the whole hospital stay with OD $>3.00$ measured with all samples collected from day 1 to day 15 . Positive results were also obtained using a modified in-house ELISA in which the wells are only coated by PF $4{ }^{8}$ demonstrating the presence of IgG antibodies that bound PF4 alone (data not shown). Such characteristics of VITT antibodies, shared with those of highly pathogenic auto-immune heparin-induced thrombocytopenia (HIT) antibodies, ${ }^{9}$ indicate a different specificity and affinity towards PF4 compared to classical HIT antibodies, and explain why their detection by HIT-dedicated immunoassays may be inadequate. ${ }^{7}$ The fact that anti-PF4 IgG antibodies were detected using a $\mathrm{PF} 4$ /polyvinylsulfonate rapid assay (HemosIL ${ }^{\circledR}$ AcuStar HIT IgG assay) is particular since this assay failed to detect anti-PF4 antibodies in most of the reported VITT cases. Nevertheless, the HemosIL ${ }^{\circledR}$ Acustar HIT IgG assay rarely gives weak positive results in patients with likely VITT diagnosis, as stated by Platton et al., who reported two positive results out of 31 patients, all of whom had anti-PF4 IgG detected by ELISA. ${ }^{10}$

PF4 serotonin-release assay (PF4-SRA) was performed with the same samples, as previously described. ${ }^{7}$ Platelet activation, measured through maximal serotonin release, was $90 \%$ in the absence of heparin on day 1 , decreased by more than $50 \%$ after IVIg administration (day 4 , 
$35 \%$ ) and remained low when the patient was getting better (day 7, 36\%). In contrast, platelet activation returned to a high level at the time of clinical deterioration, day $15(79 \%)$. Time related changes of total IgG plasma levels mirrored those of PF4-SRA (Figure 1). While the total IgG level was rather low at diagnosis, it rose above $30 \mathrm{~g} / \mathrm{L}$ after IVIg administration and then rapidly decreased, as observed on day 15 , with a return to normal values for a healthy adult. These data support the hypothesis that the rebound of platelet activation observed in PF4-SRA at the time of clinical deterioration could be due to a rapid elimination of the infused immunoglobulins and the loss of their competing effect with platelet activating anti-PF4 antibodies on platelet FcyRIIa.

Additional experiments were performed with PF4-SRA to consolidate our hypothesis that the levels of IgG can explain the exacerbation The same samples were diluted either in normal plasma (containing normal IgG level) or in modified Tyrode's buffer at 1/10, 1/20, 1/40 dilution ratios. When samples were diluted in normal plasma, platelet activation decreased proportionally with the dilution, and was completely abolished at $1 / 40$, except for the day 1 sample, which still slightly activate platelets under these conditions (Figure 2). In contrast, when the samples were diluted in Tyrode's buffer platelet activation remained high (near $100 \%$ ) for the samples collected on day 1 and day 15 but increased for those obtained at days 5 and 7, approaching 100\% (Figure 2). These results strongly support that platelet activation by VITT antibodies was inhibited by normal $\mathrm{IgG}$, and that lowering the concentrations of normal IgG led to the reappearance of platelet activation by loss of competition between the IVIg and anti-PF4 IgG.

This case supports the concept that proper monitoring using an appropriate functional assay could help in the clinical decision making since PF4-SRA mirrored with the clinical evolution of the patient. Such an observation has also been made in a recent study, which demonstrated that platelet activation by VITT antibodies was inhibited in patients treated with IVIg. ${ }^{11}$ Nevertheless, this needs to be confirmed. Interestingly, the inhibitory effect of normal polyclonal IgG on the platelet activation induced by PF4-specific antibodies could also vary from one patient to another, as previously demonstrated in HIT patients. ${ }^{12}$ It is also important to note that administration of IVIg reduces the activation of platelets as assessed by the PF4-SRA. This has major consequences when collecting samples for confirmation of VITT diagnosis and dilution in appropriate buffer, as we did in our experiments, could be recommended to assess the competitive interaction between anti-PF4 IgG and IVIg. However, this test lacks worldwide availability, and cannot easily be used for emergency patient monitoring. Therefore, as total IgG concentration measured in the patient inversely correlated with platelet activation in PF4-SRA, quantitatively assaying anti-PF4 IgG antibodies levels and total IgG concentration in the patient's plasma could help to identify situations where the competition between normal polyclonal IgG and anti-PF4 IgG on Fc $\gamma$ RIIa may switch in favor of the platelet activating antibodies. ${ }^{13}$

Even though data on IVIg clearance parameters and target concentrations are lacking for such a very peculiar condition, a rapid decrease in total IgG concentrations within the normal range (i.e., 7-16 g/L) ${ }^{14}$ could alert to possible therapeutic escape, and the need for re-administration of IVIg, especially in a situation where anti-PF4 $\mathrm{IgG}$ remains high. In the patient, total IgG concentration was reduced by half within 8 days, which is substantially faster than the median half-life of 30 days generally reported in the literature for IVIg. ${ }^{15}$ Although further studies are needed to understand the accelerated clearance and to assess the clinical relevance of total IgG measurement to monitor the efficacy of IVIg, it appears a very affordable tool in medical practice in combination with anti-PF4 IgG antibodies testing.

\section{Jonathan Douxfils, ${ }^{1,2^{*}}$ Caroline Vayne, ${ }^{3 *}$ Claire Pouplard, ${ }^{3}$} Thomas Lecompte, ${ }^{4}$ Julien Favresse, ${ }^{1,5}$ Florence Potier, ${ }^{6}$ Emy Gasser, ${ }^{7}$ Valérie Mathieux, ${ }^{8}$ Jean-Michel Dogné, ${ }^{1}$ Yves Gruel, Jérôme Rollin ${ }^{3 \#}$ and François Mullier ${ }^{3 \#}$

'University of Namur, Department of Pharmacy, Namur Research for Life Sciences, Namur Thrombosis and Hemostasis Center, Namur, Belgium; ${ }^{2}$ QUALIblood s.a., Namur, Belgium; ${ }^{3}$ University of Tours, EA7501 GICC, CHRU de Tours, Department of Hemostasis, Tours, France; ${ }^{4}$ Département de Médecine, Hôpitaux Universitaires de Genève, Service d'Angiologie et d'Hémostase et Faculté de Médecine, Geneva Platelet Group (GpG), Université de Genève, Geneva, Switzerland; ${ }^{5}$ Clinique Saint-Luc Bouge, Department of Laboratory Medicine, Bouge, Belgium; 'Service de Gériatrie, CHU UCL Namur site Sainte-Elisabeth, Namur, Belgium; ' Université Catholique de Louvain, Service de Gériatrie, CHU UCL Namur site SainteElisabeth, Namur, Belgium; ${ }^{8}$ CHU UCL Namur Isite SainteElizabeth, Université Catholique de Louvain, Department of Hematology, Namur Research Institute for Life Sciences, Namur Thrombosis and Hemostasis Center, Yvoir, Belgium and "Université Catholique de Louvain, CHU UCL Namur, Namur Thrombosis and Hemostasis Center, Hematology Laboratory, Namur Research Institute for Life Sciences, Yvoir, Belgium.

\section{*JD and CV contributed equally as co-first authors \\ \#R and FM contributed equally as co-senior authors Correspondence: JONATHAN DOUXFILS -}

jonathan.douxfils@unamur.be

doi:10.3324/haematol.2021.279509

Received: June 28, 2021.

Accepted: August 3, 2021.

Pre-published: August 12, 2021

Disclosures: JD is the CEO and founder of QUALIblood S.A., a contract research organization manufacturing the DP-Filter, is a co-inventor of the DP-Filter (patent application number: PCT/ET2019/ 052903) and reports personal fees from Daiichi-Sankyo, DOASense Gmbh, Gedeon Richter, Mithra Pharmaceuticals, Norgine, Portola, Stago, Roche and Roche Diagnostics outside the submitted work; TL reports non-personal fees from IRIS and Stago; FM reports institutional fees from Stago, Werfen, Nodia, Roche Sysmex and Bayer as well as speaker fees from Boehringer Ingelheim, Bayer Healthcare, Bristol-Myers Squibb-Pfizer, Stago, Sysmex and Aspen all outside the submitted work. The other authors have no conflicts of interest to disclose.

Contributions: JD and $C V$ analyzed the results, wrote the first draft of the manuscript and designed the figures; JR, YG, CP, CV provided and analyzed the results and revised the manuscript; TL analyzed the results and thoroughly revised the manuscript; JF provided and analyzed the results and revised the manuscript; FP, EG and VM managed the patient and thoroughly revised the manuscript; J-MD analyzed the results; FM designed and supervised the experiments, provided and analyzed the data, and interpreted the results.

Acknowledgments: the authors would like to thank the technical staff of the CHU UCL Namur, Mrs Justine Baudar, Mrs Maité Guldenpfennig and the technical staff of the CHRU Tours, Mrs Séverine Augereau.

Informed consent and ethical committee approval: as the patient died, an independent Review Board of the Clinique Sainte-Elisabeth 
CHU UCL Namur (OMO70) was consulted to decide if reporting this case is ethically acceptable. This Ethical Committee (decision number: 27-21) was in favor of the publication of this case and did not see any ethical issue in publishing this case report. All data has been anonymized.

\section{References}

1. Morel-Kopp MC, Mullier F, Gkalea V, et al. Heparin-induced multielectrode aggregometry method for heparin-induced thrombocytopenia testing: communication from the SSC of the ISTH. I Thromb Haemost. 2016;14(12):2548-2552.

2. 2. Robert T. Proposed Brighton Collaboration process for developing a standard case definition for study of new clinical syndrome $\mathrm{X}$, as applied to thrombosis with thrombocytopenia syndrome (TTS) V10.16.3. Accessed on May 30, 2021. Available from: https://brightoncollaboration. us / w pcontent/uploads/2021/04/TTS-Case-Finding-and-DefinitionProcess.v9.0-April-16-202115853.pdf.

3. 3. Guidance produced from the Expert Hematology Panel (EHP) focused on Covid-19 vaccine induced thrombosis and thrombocytopenia (VITT) updated guidance on management. Version 1.3. Accessed on Apr 7, 2021. Available from: https://b-sh.org.uk/media/19530/guidance-version-13-on-mngmt-of-thrombosis-with-thrombocytopenia-occurring-after-c-19vaccine_20210407.pdf.

4. Rosenberger LH, Smith PW, Sawyer RG, et al. Bilateral adrenal hemorrhage: the unrecognized cause of hemodynamic collapse associated with heparin-induced thrombocytopenia. Crit Care Med. 2011;39(4):833-838.

5. Scully M, Singh D, Lown R, et al. Pathologic antibodies to platelet factor 4 after ChAdOx1 nCoV-19 vaccination. N Engl J Med.
2021;384(23):2202-2211.

6. Bussel JB, Connors J, Cines DB, et al. Thrombosis with thrombocytopenia syndrome (also termed vaccine-induced thrombotic thrombocytopenia). version 1.5. Accessed on Jul 26, 2021. Available from: https://www.hematology.org/covid-19/vaccine-induced-immunethrombotic-thrombocytopenia.

7. Vayne C, Rollin J, Gruel Y, et al. PF4 Immunoassays in vaccineinduced thrombotic thrombocytopenia. N Engl J Med. 2021; 385(4):376-378

8. Pouplard C, Leroux D, Rollin J, et al. Incidence of antibodies to protamine sulfate/heparin complexes incardiac surgery patients and impact on platelet activation and clinical outcome. Thromb Haemost. 2013;109(6):1141-1147.

9. Nguyen T-H, Medvedev N, Delcea M, Greinacher A. Anti-platelet factor 4/polyanion antibodies mediate a new mechanism of autoimmunity. Nat Commun. 2017;8:14945.

10. Platton S, Bartlett A, MacCallum P, et al. Evaluation of laboratory assays for anti-platelet factor 4 antibodies after ChAdOx1 nCOV-19 vaccination. J Thromb Haemost. 2021;19(8):2007-2013.

11.Bourguignon A, Arnold DM, Warkentin TE, et al. Adjunct immune globulin for vaccine-induced thrombotic thrombocytopenia. N Engl J Med. 2021;385(8):720-728.

12. Rollin J, Pouplard C, Sung HC, et al. Increased risk of thrombosis in FcgammaRIIA 131RR patients with HIT due to defective control of platelet activation by plasma IgG2. Blood. 2015;125(15):2397-2404.

13. Warkentin TE. High-dose intravenous immunoglobulin for the treatment and prevention of heparin-induced thrombocytopenia: a review. Expert Rev Hematol. 2019;12(8):685-698.

14. Svendsen PJ, Ward AM, Dati F, et al. New international reference preparation for proteins in human serum (RPPHS). Clin Chem. 1994:40(6):934-938

15.Koleba T, Ensom MH. Pharmacokinetics of intravenous immunoglobulin: a systematic review. Pharmacotherapy. 2006; 26(6):813-827. 